\title{
Zur Erosionsgefährdung von brachgelegten Wiesen und Weiden im Berggebiet
}

\section{Alpine Erosions- und Rutschvorgänge}

Vor der Inangriffnahme des Themas «Prozesse der Erosionsentwicklung auf brachgelegten Bergwiesen und Alpweiden der subalpinen Stufe» erscheint es zweckmäßig, diese sehr speziellen Abtragsformen zunächst ganz kurz in den Rahmen der im alpinen Raum zu beobachtenden Massenbewegungen und Massenselbstbewegungen zu stellen.

Dabei kann auch ein Einblick in den Stellenwert gewonnen werden, den brachebedingte Bodenabträge im Gesamtrahmen alpiner Massenabträge einnehmen. Im folgenden wird von deren Gliederung nach BUNZA et al. (1976) ausgegangen, wobei Abträge durch oberflächlich anströmendes Wasser oder durch Schneeschurf, Massenbewegungen und Rutschvorgänge unterschieden werden. Letztere werden durch Instabilitäten in Hängen als Folge der Schwerkraft meist unter Mitwirkung von Wasser verursacht.

Erosive Angriffe durch strömendes Wasser in Form von Uferanbrüchen und Feilenanbrüchen sind hier nicht näher zu betrachten; auch Bodenverletzungen durch Schneeschurf, insbesondere durch Lawinen sind mit brachgefallenem Grünland nicht ursächlich in Zusammenhang zu bringen. Der Grund dafür ist sehr einfach darin zu sehen, daß jede Rasenvegetation, gleich unter welcher Bewirtschaftung oder ehemaligen Bewirtschaftung an exponierten Stellen, insbesondere an konvexen Hangpartien von stürzenden Schneemassen durch Schurf zerstört werden kann. Dies gilt insbesondere bei Naßschneelawinen und Schneebrettern.

Schäden dieser Art lassen sich im Gelände meist gut erkennen, da sie vielfach striemenartige Schurfspuren in Hangrichtung aufweisen und weil ihre hangaufwärts gelegenen Begrenzungen flach abgehobelt und nicht als Abbruchkante ausgebildet sind. Diese Abtragsformen werden im folgenden nicht weiter behandelt. Sie sind nur deshalb erwähnt, weil sie nicht selten mit Bodenschäden als Folge von Rutschvorgängen verwechselt werden, die unter Umständen mit dem Brachfallen von Grünland in Zusammenhang gebracht werden müssen.

Bei den Rutschungen sind zwei große Gruppen zu unterscheiden. Bei der einen, den fast nur in Lockergesteinen auftretenden Rotationsrutschungen, entsteht die Gleitfläche erst unmittelbar während der Rutschung. Ihr Umfang und die Tiefenlage der
Gleitbahn hängen von einer Reihe von Umständen $a b$, die konstant oder variabel sind. Von den konstanten Größen seien genannt Relief, Korngrößenverteilung, Porenvolumen und Porenverteilung; die Tiefenlage der Gleitbahn hängt vielfach von der witterungsbedingt unterschiedlichen Durchlässigkeit des Lockergesteinskörpers zum Zeitpunkt des auslösenden Niederschlagsereignisses ab. Diese Rotationsrutschungen treten im Zusammenhang mit Starkregenereignissen, insbesondere in periglazialen Talverfüllungen und in frühpostglazialen Hangschuttkörpern auf. Die bewegten Massen können sehr umfangreich sein und führen nicht selten im Zusammenhang mit Hochwasserereignissen in Wildbächen zu katastrophalen Vermurungen in den Tälern. Es sei in diesem Zusammenhang an die Ereignisse 1966 in Kleindorf in Kärnten, 1969 in Inzing in Nordtirol und an die Wildbachlandschaften in den Talverfüllungen der Nordalpen zwischen Lech und Isar erinnert (KARL und DANZ 1969). Mit Brache sind die Rotationsrutschungen allerdings nicht in ursächlichen Zusammenhang zu bringen.

Die nächste große Gruppe von Rutschungen sind die Translationsrutschungen, denen gemeinsam ist, da $\beta$ sich der Rutschvorgang auf vorgeformten Gleitflächen abspielt.

Diese Gleitflächen können tektonisch oder lithologisch bedingt sein, sie können aber auch bodengenetisch entstanden sein. Rutschauslösendes Agens ist Wasser, wenn man von seismischen Erschütterungen absieht, die insbesondere bei tixotropen Gleitschichten Translationsrutschungen auslösen können.

In dieser Gruppe der Translationsrutschungen finden sich in Umfang wie Aussehen sehr unterschiedliche Formen, im Gegensatz zu den im Erscheinungsbild doch recht einheitlichen Rotationsrutschungen.

Ähnlich wie bei den Talzuschüben und Bergzerreißungen meist riesigen Ausmaßes können auch bei Translationsrutschungen mit weniger tiefliegenden Gleitflächen ganze Hänge in Bewegung geraten.

Räumlich kleinere bis kleine Translationsrutschungen finden entweder unmittelbar an der Grenzfläche zwischen Boden und anstehendem Gestein statt, wie

Dr. J. Karl, Regierungsdirektor Bayer. Amt für Wasserwirtschaft Prinzregentenstr. 24, D-8000 München 19 
dies bei den Waldabbrüchen der Fall ist, wie sie MAYER und BEINSTEINER aus Osttirol beschrieben haben, oder im Boden selbst, bei den Translationsbodenrutschungen.

Die Talzuschübe und Bergzerreißungen spielen bei unserer Betrachtung keine Rolle, wohl aber ausgedehnte Translationsrutschungen in Lockergesteinen oder in veränderlichfesten Gesteinen und Translationsbodenrutschungen.

\section{Translationsrutschungen als Folge von Brachen}

BUNZA und der Verf. konnten solche großräumigen Rutschvorgänge in Corvara in den Südtiroler Dolomiten untersuchen und fanden dabei deutliche Zusammenhänge mit dem Brachfallen dieser Hänge (BUNZA 1976, 1978).

Die Landschaft um Corvara wird von zwei Gruppen von Großformen beherrscht: Da sind einmal die Dolomitklötze mit ihren schroffen Wänden und, in deutlichem Gegensatz dazu, weiche, mittelgebirgsartige Formen, die aus veränderlichfesten, insbedere triassischen und vulkanisch beeinflußten Mergeln und mergeligen Kalken aufgebaut sind.

Diese veränderlichfesten Gesteine sind tiefgründig verwittert, die Böden sind meist mächtige Hanggleye unterschiedlicher Ausbildung. Sowohl die Böden wie die Schuttkörper sind zumindest stellenweise stark vernäßt, die Vergetation besteht ausschließlich aus Rasengesellschaften, meist Milchkrautweiden und Borstgrasrasen. Lediglich an den steileren und etwas trockeneren Oberhängen sind kleinflächig Waldreste vorhanden, ansonsten beschränkt sich der Baumwuchs auf einige einzelstehende Lärchen.

Die Hänge wurden bis vor etwa vier Jahrzehnten regelmäßig gemäht, in Teilbereichen wohl auch beweidet. Sie waren die Lebensgrundlage der Bewohner dieses Hochtales und wurden dementsprechend gepflegt. Dazu gehörte vor allem die Unterhaltung eines Entwässerungssystems, das in Form offener Gräben die Böden einigermaßen trocken hielt und das Eindringen größerer Wassermassen in die Schuttkörper verhinderte.

In den fünfziger Jahren verfielen diese Entwässerungseinrichtungen. Die Folge sind starke Vernässungen der Schuttkörper wie der Böden und Rutschungen, die nunmehr stellenweise auch die Tallagen bedrohen. Während in den meisten Fällen beim Brachfallen von alpinem Grünland die Veränderungen der Vegetation im Vordergrund stehen, ist hier ein eher sekundär zu nennender Effekt für die Entstehung von Rutschungen wirksam: Es ist dies das wegen der fehlenden Entwässerung zusätzlich in den Untergrund eindringende Niederschlagswasser.

Dazu sind einige Bemerkungen zum Verhalten des Niederschlagswassers zumindest bei Starkregen in Rasengesellschaften angebracht.
Wie sich in einer Vielzahl von Beregnungsversuchen zeigte (KARL 1983), fließt in alpinen Rasengesellschaften mindestens ein Drittel, in manchen Fällen bis zu zwei Drittel des Niederschlages rasch oberflächlich ab. Dies würde dafür sprechen, daß Rasengesellschaften im Gegensatz zu Wald, in dem nahezu alle Niederschläge versickern, die Vernässung des Untergrundes eher verhindern als fördern. Dem ist entgegenzuhalten, daß dies nur bei Starkregen gilt. Bei schwächeren Regen ist die Interception bei Rasen wesentlich geringer als in Wäldern. Das gleiche gilt für die Evapotranspiration. Die durch Versickerung in den Untergrund gelangende Wassermenge ist dementsprechend in Rasengesellschaften wesentlich größer als in Wäldern. Das heißt, daß die Scherfestigkeit des Schuttkörpers langfristig nahe an den Punkt herangeführt wird, an dem der Winkel der inneren Reibung auf Null absinkt und es damit zur Rutschung kommt (BENDEL 1949). Da die Annäherung an diesen Punkt zwar allmählich stattfindet, die Rutschung bei seinem Erreichen jedoch abrupt eintritt, genügen letztlich ganz geringe zusätzliche Wassermengen, um die Rutschung auszulösen. Die alten Entwässerungssysteme waren offenbar in der Lage, diese entscheidenden letzten Tropfen, die das $\mathrm{Fa} B$ zum Überlaufen bringen, über längere Zeiträume vom Untergrund fernzuhalten. Dies war nach ihrem Verfall nicht mehr gegeben.

Die Sanierung dieser Hänge wurde aufgrund dieser Zusammenhänge in Form von Entwässerungen der Oberfläche und der zahlreichen durch Rutschmassen aufgestauten Tümpel in Angriff genommen, nachdem mit Wirtschaftswegen die Wiesen für heute übliche Geräte zugänglich gemacht wurden.

\section{Translationsbodenrutschungen als Folge von Brache}

War in Corvara die Zusammensetzung der Rasenvegetation von untergeordneter Bedeutung, so ist diese bei den nunmehr zu behandelnden Translationsbodenrutschungen ausschlaggebend. Doch zunächst zum Begriff: Unter Translationsbodenrutschungen werden solche Massenbewegungen verstanden, bei denen sich innerhalb der meist mächtigen Böden ein Gleithorizont bildet, auf dem die Rasendecke abgleitet (BUNZA 1976). Voraussetzung ist ein für alpine Verhältnisse mächtig entwickeltes Bodenprofil und eine gewisse Steilheit des Hanges.

Es erhebt sich hier zunächst die Frage, wie die für die Entstehung eines Gleithorizontes im Boden notwendigen Wassermengen in den Boden kommen können, wenn auf alpinen Rasengesellschaften $40 \%$ der Niederschläge oder mehr zumindest bei Starkregen oberflächlich abfließen.

Die Antwort darauf ergibt sich, wenn die Entstehung solcher Translationsbodenrutschungen näher betrachtet wird. 
Dazu ist allerdings ein Blick auf die Entwicklung der Vegetation nach dem Aufhören der Nutzung zu werfen.

Die nach der Rodung von sehr unterschiedlichen Waldgesellschaften und von Grünerlengebüschen entstandenen Rasengesellschaften waren unter jahrhundertelanger Mäh- und teilweise auch Weidenutzung zu, pflanzensoziologisch gesehen, ausgeglichenen Pflanzengesellschaften geworden. SCHAUER (1975) hat dies im Rahmen seiner Untersuchungen über Translationsbodenrutschungen schematisch dargestellt und betrachtet als solche Sekundärrasen unterhalb der potentiellen Waldgrenze BlaugrasHorstseggenrasen, Goldhaferwiesen, Violettschwingelrasen, Alpenfettweiden, Rostseggenrasen und Borstgrasrasen.

Nach Aufhören der Nutzung entwickeln sich solche Rasen vielfach zu Pflanzengesellschaften, die sich nicht ohne weiteres in pflanzensoziologische Systeme einordnen lassen.

Diese gestörten Pflanzenverbände zeichnen sich oft durch verstärktes Auftreten einer oder weniger Arten aus, wie etwa von Deschampsia caespitosa, der Rasenschmiele, oder von Dactylis glomerata, dem Knaulgras. Auch Nardus stricta, das Borstgras, kann sich unter Umständen zusammen mit den genannten Arten stark entwickeln.

Untersucht man, in welchen Pflanzengesellschaften Translationsbodenrutschungen gehäuft auftreten, so ist festzustellen, daß dies in solchen gestörten, stark mit horstbildenden Gräsern durchsetzten Rasen der Fall ist.

Dies trifft insbesondere dann zu, wenn die Hangneigung $30^{\circ}$ übertrifft.

Die Voraussetzungen für Translationsbodenrutschungen an solchen Hängen entstehen im Winter. Schneedecken unterliegen vielfach Kriechbewegungen, die auch oberflächlich als Zugrisse erkennbar sind.

Solche Kriechbewegungen einer Schneedecke können gewissermaßen ungestört ablaufen, wenn die Bindung des Schnees an den Untergrund nur schwach ist, beispielsweise auf einer Lahnergras-Vegetation aus Calamagrostis varia oder Avena parlatorei.

Sind in der Rasendecke jedoch horstbildende Arten wie Deschampsia caespitosa oder Dactylis glomerata vorhanden, so frieren diese Horste in die Schneedecke ein, und die Zugkräfte der kriechenden Schneedecke werden auf den Boden übertragen. Es entstehen nicht nur Zugrisse in der Schneedecke, sondern auch im Boden. Halbmondförmige, bis etwa 20 Meter lange und nur wenige Zentimeter breite Risse sind in der Rasendecke nach der Schneeschmelze vielfach $\mathrm{zu}$ beobachten.

Auf weidegenutzten Hängen entstehen solche Risse ebenfalls gelegentlich, werden jedoch wieder zugetreten, da das opportunistisch vorgehende Weidevieh diese schmalen Hangleisten als Gangsteige benutzt. Auf ungenutzten Hängen bleiben sie nicht nur offen, sie entstehen auch viel häufiger, da die zahlreichen ungemähten oder nicht abgeweideten Grashorste vermehrt Ansatzpunkte für die kriechende Schneedecke bieten. In diese Zugrisse im Boden fließt bei Starkregen das in großer Menge abfließende Oberflächenwasser. Es sucht sich als interflow, als oberflächennaher Abfluß im Boden einen Weg und findet ihn dort, wo die Mehrzahl der Graswurzeln endet, das Bodenprofil jedoch noch nicht zum Anstehenden übergeht.

Wie Korngrößenanalysen zeigten, ist der Schluffanteil in diesem Bereich deutlich höher als in den nicht oder nicht mehr durchwurzelten Bodenschichten. Es ist aus einer Vielzahl von Untersuchungen über Rutschungen und erosive Vorgänge bekannt, daß ein hoher Schluffanteil stark zur Erosionsanfälligkeit und Instabilität beiträgt. Schluffreiche Böden werden dementsprechend meist sehr stark von der Erosion ergriffen, und schluffreiche Lockergesteine und Böden neigen mehr zu Rutschungen als andere.

In unseren Fällen bewegen sich die Schluffanteile in der von der Rutschung ergriffenen Bodenschicht zwischen 19,8 und 79,3 Gewichtsprozenten, wobei das Mittel aus 23 Proben bei 49,9\% liegt (sCHAUER 1975).

Vor einer Betrachtung der Wirkung des interflow im Boden ist ein weiterer kurzer vegetationskundlicher Exkurs notwendig.

In vielen der als Folge der Brachlegung gestörten Rasen fallen wahrscheinlich aus Konkurrenzgründen zahlreiche Stauden, insbesondere Umbelliferen und Leguminosen aus. Viele dieser Arten besitzen kräftige Pfahlwurzeln, die die meist mächtigen Böden bis zu Tiefen von 60 Zentimetern und mehr durchwurzeln. SCHAUER (1975) hat in einer Reihe von Wurzelprofilen dargestellt, daß die in den gestörten Vegetationskomplexen überwiegenden Gräser nur Durchwurzelungstiefen von 20 bis 30 Zentimetern erreichen. Am deutlichsten ist dies bei Nardus stricta, das bis zu dieser Tiefe einen dichten Wurzelfilz ausbildet, in die darunterliegenden Bodenschichten jedoch nicht eindringt.

Auf diesem Grenzhorizont zwischen durchwurzeltem und nicht durchwurzeltem Boden findet das durch die Zugrisse eingedrungene Wasser einen Weg und wegen des hohen Schluffanteiles auch ein Medium, das zur Bildung von Gleithorizonten prädestiniert ist.

Mangelnde Haftung an den Untergrund, steile Hanglage, eine Bodenschicht mit geringer innerer Reibung führen zusammen mit einer durch Wassersättigung schwer gewordenen Rasendecke zum Abgleiten; eine Blaike oder besser eine Translationsbodenrutschung ist entstanden. Wie weit die ausgebrochene Rasenscholle abgleitet, ist mehr zufälliger Natur. Sie kann sich aufrollen oder nur wenig abgleiten, sie kann aber auch bis zum Hangfuß abfahren.

Es ist vielfach zu beobachten, daß es ausgesprochene Blaikenjahre gibt und dann wieder Jahre, in denen solche Rutschungen fast nicht beobachtet werden. Aus der Entstehung der Translationsbodenrutschungen läßt sich dieses Phänomen unschwer deuten, 
wenn auch noch nicht alle Zusammenhänge bis ins einzelne geklärt sind.

Voraussetzung ist eine mächtige Schneedecke, die aufgrund ihres Aufbaues zu Kriechbewegungen neigt. Sind so einmal die Zugrisse im Boden geschaffen, dann bedarf es eines oder mehrerer sommerlicher Starkregen, um die Rasenschollen zum Abgleiten zu bringen. Es sind also zwei jeweils jahreszeitlich gebundene Voraussetzungen, die zu Massenschäden dieser Art führen.

Die Entwicklung der so von Vegetation entblößten Flächen geht vielfach über Pioniergesellschaften zu relativ artenarmen Rasengesellschaften, in denen auch Moose und Flechten einen größeren Anteil haben können. Auf armen, oberflächlich versauerten Böden können sich auch moos- und flechtenreiche Zwergstrauchheiden entwickeln.

Größere sekundäre Feststoffausträge sind aus diesen durch Translationsbodenrutschungen entstandenen Flächen nicht $\mathrm{zu}$ erwarten. Ihre Bedeutung als Feststoffherde im Feststoffregime der Wildbäche ist daher gering, zumal die feinkörnigen Böden als Schwebstoffe schadlos abgeführt werden.

Bei der Gewichtung der Gefährdungsfaktoren für die Ausscheidung von Problemgebieten stehen die Gesteine und die Hangneigung im Vordergrund. In zweiter Linie sind Ausgangsvegetation sowie Klima und Witterung zu betrachten.

\section{Prognosen}

Prädestiniert für großflächige, meist auch tiefergreifende Translationsrutschungen sind veränderlichfeste Gesteine im Sinne von KEIL (1959) dann, wenn ihre mächtigen frühpostglazialen Schuttmäntel zur Vernässung neigen. $\mathrm{Zu}$ nennen sind hier beispielsweise Mergel des Flysch wie des Ultrahelvetikums, Bündnerschiefer, Allenien-Schichten, Buchensteiner Schichten.

Translationsbodenrutschungen treten in mächtigen Bodenbildungen aus veränderlichfesten Gesteinen auf. Hier sind zu nennen die Zementmergel-Kieselkalkserie des Flysch, die Allgäuschichten des Lias, auch Lias-Fleckenmergel genannt, darüber hinaus aber auch andere veränderlichfeste Gesteine, wie Raibler- oder Kössenerschichten, wenn sie mächtige, nicht oder nur schwach vernäßte Böden entwickelt haben.

Zur Hangneigung ist zu bemerken, daß Translationsbodenrutschungen kaum unter $30^{\circ}$ Neigung auftreten. Es sind also recht steile Hänge, die betroffen sind.

Das Kleinrelief darf in diesem Zusammenhang nicht außer acht bleiben. Durch Viehgangeln getreppte Hänge sind weit weniger gefährdet als glatte Hänge, da die Kleinterrassen der Gangel das Schneekriechen verhindern.

Die Ausgangsvegetation ist insofern von Bedeutung, als insbesondere solche Vegetationseinheiten gefähr- det sind, die in größerem Umfang horstbildende Gräser enthalten.

Klimatisch und witterungsmäßig sind hohe Schneelagen mit Neigung zu Kriechvorgängen und sommerliche Starkregen die wichtigste Voraussetzung für die Entstehung von Translationsbodenrutschungen.

Prognosen lassen sich unter diesen Bedingungen nur sehr generell stellen. Grundlagen dafür sind geologische und topographische Karten, aus denen die veränderlichfesten Gesteine und die Hangneigungen zu entnehmen sind. Des weiteren sind Karten maximaler Schneehöhen und sommerlicher Starkregenhäufigkeit von Nutzen. Wann und wo dann im einzelnen Rutschungen auftreten, läßt sich allerdings nicht voraussagen.

\section{Künftige Forschungsaufgaben}

Fragen für die künftige Forschung sind in erster Linie in der Aufklärung der Bodenentwicklung nach dem Aufhören der Nutzung und in der Abklärung der Bodenmechanik in der gleitanfälligen Bodenschicht zu sehen.

Des weiteren wären die für die Entstehung von Kriechschneebewegungen verantwortlichen witterungsmäßigen Umstände zu erkunden, beispielsweise der Frostzustand des Bodens beim Zuschneien eines Hanges. Auch die metamorphen und diagenetischen Vorgänge in der Schneedecke sind sicherlich für die Entstehung von Kriechbewegungen von erheblichem Interesse. Bei den großflächigen Translationsrutschungen im Zusammenhang mit Vernässungen wären bodenmechanische Untersuchungen und Versuche über das Verhalten von Niederschlägen auf oberflächlich entwässerten und aus brachgefallenen, sekundär vernäßten Hängen notwendig.

\section{Zusammenfassung}

Translationsrutschungen und Translationsbodenrutschungen können direkt oder indirekt durch Brachfallen von Grünland in der montanen und subalpinen Stufe entstehen.

Am Beispiel Corvara werden tiefgreifende Translationsrutschungen in vernäßten Schuttkörpern aus veränderlich-festen Gesteinen als Folge des Verfalls alter Entwässerungseinrichtungen beschrieben.

Translationsbodenrutschungen entstehen in brachgefallenem Grünland vor allem in der subalpinen Stufe an Steilhängen durch Veränderungen in der Vegetationsdecke. Sie führen dazu, daß als Folge der winterlichen Schneeauflast Zugrisse im Boden entstehen. Bei sommerlichen Starkregen dringt Oberflächenwasser in diese Risse ein. Auf den so entstehenden Gleithorizonten gleiten die Rasenschollen ab. 


\section{Literatur}

BEINSTEINER, H., MAYER, H. (1971): Waldbauliche Beurteilung von Waldabbrüchen im Osttiroler Katastrophengebiet. Diss. Hochschule für Bodenkultur, Wien

BENDEL, L. (1949): Ingenieurgeologie. Wien

BUNZA, G. (1976): Translationsrutschungen in Corvara. Unveröff. Gutachten

BUNZA, G. (1978): Bewegungsablauf und Sanierungsmöglichkeiten von Erdströmen. In: Geol. Paläont. Mitt. Innsbruck, Band 8, Festschrift W. HEISSEL, S. 209-225

BUNZA, G., KARL, J., MANGELSDORF, J., SIMMERSBACH, P. (1976): Geologisch-morphologische Grundlagen der Wildbachkunde. Schriftenreihe des Bayer. Landesamtes für Wasserwirtschaft, H. 17, 2. unveränderte Auflage 1982
KARL, J. (1961): Blaikenbildung auf Allgäuer Blumenbergen. Jahrb. d. Vereins z. Schutz d. Alpenpflanzen u. -tiere 26

KARL, J. (1983): 10 Jahre AbfluB- und Erosionsmessungen auf land- und forstwirtschaftlichen Nutzflächen mit Hilfe künstlicher Starkregen. Bayerisches Landesamt für Wasserwirtschaft, Informationsbericht $1 / 83$

KARL, J., DANZ, W. (1969): Der Einfluß des Menschen auf die Erosion im Bergland. Schriftenreihe der Bayer. Landesstelle für Gewässerkunde, H. 1

KEIL, K.-F. (1959): Geotechnik. Halle (Saale)

SCHAUER, Th. (1975): Die Blaikenbildung in den Alpen. Schriftenreihe des Bayerischen Landesamtes für Wasserwirtschaft, H. 1

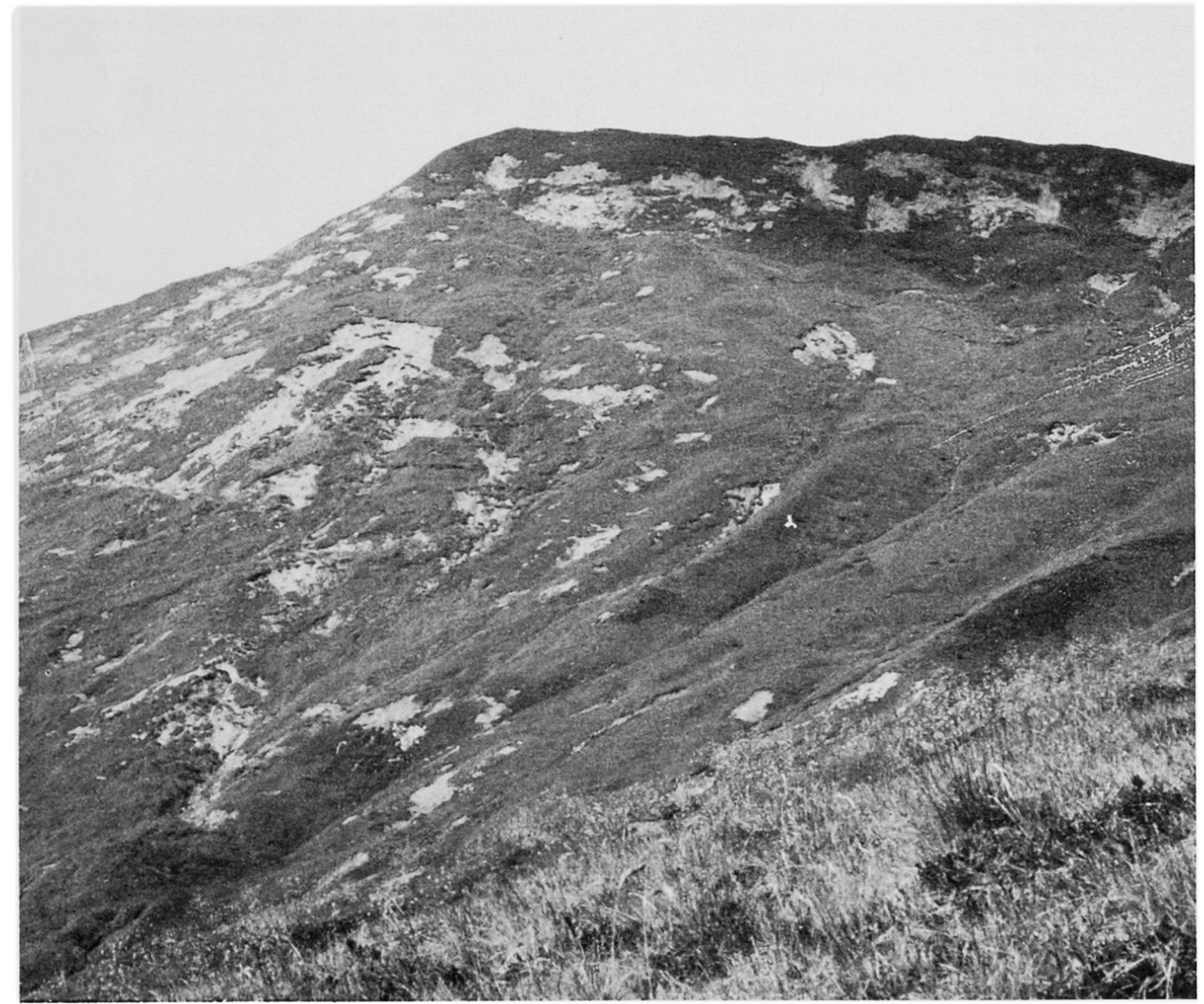

Großräumige Zunahme der Blaiken auf brachgelegten Bergwiesen im Vorderen Schanfigg (Aufnahme M. Ruf). 